\title{
A model for the static properties of DH lasers
}

\section{Buus, Jens}

\section{Published in:}

I E E E Journal of Quantum Electronics

Link to article, DOI:

10.1109/JQE.1979.1070082

Publication date:

1979

\section{Document Version}

Publisher's PDF, also known as Version of record

Link back to DTU Orbit

\section{Citation (APA):}

Buus, J. (1979). A model for the static properties of DH lasers. I E E E Journal of Quantum Electronics, 15(8), 734-739. https://doi.org/10.1109/JQE.1979.1070082

\section{General rights}

Copyright and moral rights for the publications made accessible in the public portal are retained by the authors and/or other copyright owners and it is a condition of accessing publications that users recognise and abide by the legal requirements associated with these rights.

- Users may download and print one copy of any publication from the public portal for the purpose of private study or research.

- You may not further distribute the material or use it for any profit-making activity or commercial gain

- You may freely distribute the URL identifying the publication in the public portal

If you believe that this document breaches copyright please contact us providing details, and we will remove access to the work immediately and investigate your claim 


\title{
A Model for the Static Properties of DH Lasers
}

\author{
JENS BUUS, MEMBER, IEEE
}

\begin{abstract}
The various subproblems for DH lasers such as field distribution, carrier profile, and temperature distribution are investigated. Solutions to these problems are obtained either analytically or by precise numerical methods.

By combining the subproblems, a detailed model for the static properties is obtained. The model is applicable as well below as above threshold and properties of interest in the application of DH lasers such as threshold current, field distribution at a given current, and light current characteristics can be found.

Nonlinear characteristics are found even for ideal symmetrical lasers. These "kinks" are associated with higher order modes and appear at relatively high values of the optical power.
\end{abstract}

\section{InTRODUCTION}

$\mathrm{T}$ HE DH lasers under consideration are described by the structure shown in Fig. 1.

The hatched area in Fig. 1 is the proton bombarded zone. In case of oxide insulation the thickness of the spreading layer $t_{s}$ is equal to $t_{4}$ ( $p$ layer) plus $t_{6}$ (cap).

For a given current $I$, the problem consists in finding the current density distribution $J$, the temperature $T$, the carrier density profile $N$, the intensity distributions $I_{p}$, and amplitudes $s_{p}$. The lateral modes are denoted by the mode number $p . \quad J, T, N$, and $I_{p}$ are functions of the position $y$ in the junction plane.

We start by treating the various problems independently. The method used for solving the field problem is an extension of the results from [1]-[4]. By coupling the problems a model for the laser is obtained. This model is discussed in connection with [6], [7] and some results are presented.

\section{SubProblems}

\section{A. Formulation of Field Problem}

When variations of the field distribution with length ( $z$ direction in Fig. 1) are neglected the field problem is two dimensional. The complex permittivity is a function of the $x$ and $y$ positions and is approximately given by

$$
\epsilon(x, y)= \begin{cases}\epsilon_{1}(y)=n_{1}(y)^{2}+j \frac{n_{1}(y) g_{1}(y)}{k} & |x| \leqslant t \\ \epsilon_{2}(y)=n_{2}(y)^{2}+j \frac{n_{2}(y) g_{2}(y)}{k} & |x|>t .\end{cases}
$$

$n, g$, and $k$ are the refractive index, gain, and free space propagation constant, respectively. Index 1 refers to the active layer

Manuscript received November 8, 1978; revised January 17, 1979. This work was supported by the Danish Government Fund for Scientific and Industrial Research.

The author is with the Electromagnetics Institute, Technical University of Denmark, Lyngby, Denmark.

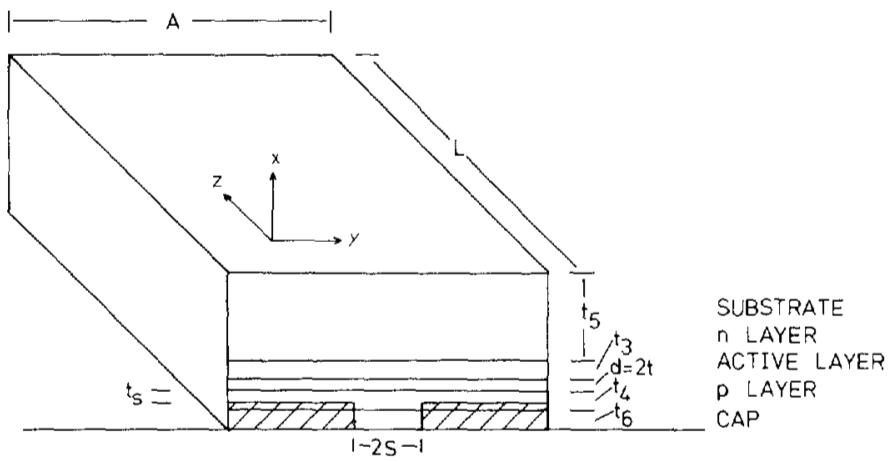

Fig. 1. Structure of DH stripe laser.

and index 2 refers to the confinement layers. In all practical cases $n \gg|g| / k$ and the approximation leading to (1) consists of neglecting $(g / 2 k)^{2}$ compared to the other terms.

The difference between the refractive indexes $n_{1}$ and $n_{2}$ is due to different aluminum content inside the active layer and in the $n$ and $p$ layers. This difference is several orders of magnitude larger than the $y$ variation of $n_{1}$ and $n_{2}$.

With $\epsilon$ given by (1) we cannot solve the field problem directly by separating the $x$ variation and the $y$ variation. Using the "effective dielectric constant," e.g., [1], however, we obtain the following equation for the $y$ variation of TE modes [2]:

$$
\frac{d^{2} y}{d y^{2}}+\left(\beta_{y}^{2}+\left(\epsilon_{\mathrm{eff}}(y)-\epsilon_{\mathrm{eff}}(0)\right) k^{2}\right) Y=0
$$

where $\beta_{y}^{2}=\Lambda_{p}, p=0,1, \cdots$ are eigenvalues and

$$
\epsilon_{\mathrm{eff}}(y)=b \epsilon_{1}(y)+(1-b) \epsilon_{2}(y) \text {. }
$$

$b$ is the normalized propagation constant.

We only consider the fundamental mode in the $x$ direction and it can be shown that $b$ can be interpreted as a field filling factor [2].

According to Schlosser [3] the difference between the imaginary parts of $\epsilon_{1}$ and $\epsilon_{2}$ gives rise to a small imaginary part of $b$ but leaves the real part unchanged. Hence, $b=b_{0}+$ $j b_{i}$ where $b_{0}$ is determined from $n_{1}, n_{2}$ and the thickness of the active layer.

Since $n_{1} \gg\left|g_{1}\right| / k, \quad n_{2} \gg\left|g_{2}\right| / k$, and $\left(n_{1}-n_{2}\right) \gg$ $\left|g_{1}-g_{2}\right| / k$ we get

$$
\begin{aligned}
\epsilon_{\mathrm{eff}}(y)= & b_{0}\left(n_{1}(y)^{2}+j \frac{n_{1}(y) g_{1}(y)}{k}\right) \\
& +\left(1-b_{0}\right)\left(n_{2}(y)^{2}+j \frac{n_{2}(y) g_{2}(y)}{k}\right) \\
& +j b_{i}(y)\left(n_{1}(y)^{2}-n_{2}(y)^{2}\right)
\end{aligned}
$$


where [3]

$$
b_{i}(y)=\frac{1}{2 k} \frac{g_{1}(y)-g_{2}(y)}{n_{1}(y)-n_{2}(y)} \frac{\left(1-b_{0}\right) v_{0} \sqrt{b_{0}}}{1+v_{0} \sqrt{b_{0}}} .
$$

$v_{0}$ is the normalized frequency which is considered constant because of the small variations of $n_{1}$ and $n_{2}$ with $y$. Hence,

$$
v_{0}=k t \sqrt{n_{1}(0)^{2}-n_{2}(0)^{2}} \text {. }
$$

The intensity filling factor $\Gamma$, which is the fraction of the intensity propagation in the active layer, can be written [2], [3]

$$
\Gamma=\frac{\int_{-t}^{t} I(x) d x}{\int_{-\infty}^{\infty} I(x) d x}=b_{0}+\frac{\left(1-b_{0}\right) v_{0} \sqrt{b_{0}}}{1+v_{0} \sqrt{b_{0}}}
$$

where $I(x)$ is the intensity distribution in the $x$ direction. When (5) and (7) are introduced in (4) we get

$$
\begin{aligned}
\epsilon_{\mathrm{eff}}(y)= & b_{0} n_{1}(y)^{2}+\left(1-b_{0}\right) n_{2}(y)^{2} \\
& +j \frac{n_{\mathrm{eff}}(0)}{k}\left(\Gamma g_{1}(y)+(1-\Gamma) g_{2}(y)\right) \\
n_{\mathrm{eff}}(0)= & \sqrt{b_{0} n_{1}(0)^{2}+\left(1-b_{0}\right) n_{2}(0)^{2}} .
\end{aligned}
$$

This expression shows that $\epsilon_{\text {eff }}$ can be considered as an average of $\epsilon_{1}$ and $\epsilon_{2}$, the real parts being weighted by $b_{0}$ but the imaginary parts by $\Gamma$. In [4] $\Gamma$ was used for both the real and the imaginary parts and in [2] $b_{0}$ was used in both cases.

The ratio of the variations in the real part of $\epsilon_{\text {eff }}$ to the imaginary part depends on $b_{0}$ and $\Gamma$ which are both functions of the normalized frequency $v_{0}$ [3] , and hence depends on the thickness of the active layer (6).

Using the assumptions that $g_{2}(y)$ is constant, $n_{1}$ varies with temperature and electron density, and $n_{2}$ varies with temperature only, (8) yields

$$
\begin{aligned}
\epsilon_{\mathrm{eff}}(y)-\epsilon_{\mathrm{eff}}(0)= & 2 b_{0} n_{1}(0) \frac{\delta n}{\delta N}(N(y)-N(0)) \\
& +2 n_{\mathrm{eff}}(0) \frac{\delta n}{\delta T}(T(y)-T(0)) \\
& +j \Gamma n_{\mathrm{eff}}(0) \frac{g_{1}(y)-g_{1}(0)}{k} .
\end{aligned}
$$

Threshold currents are almost exponentially depending on the temperature due to the temperature dependence of the gain. We write the gain for the stimulated emission as a function of temperature and electron density:

$$
\begin{aligned}
g(y) & =a(T) \cdot N(y)-b_{1} \\
a(T) & =a_{1}-a^{\prime}\left(T(y)-T^{\prime}\right) \\
a^{\prime} & =\frac{a_{1}}{T_{0}}, \quad a_{1}=a\left(T^{\prime}\right)
\end{aligned}
$$

where $a_{1}, a^{\prime}$, and $b_{1}$ are constants and $T^{\prime}$ the heat sink temperture. Equations $(10 b)-(10 c)$ are obtained by expanding an exponentially varying gain described by the characteristic temperature $T_{0}$ to the first order. The thermal variation of the gain given by (10) is consistent with the light current characteristics at various temperatures reported in [5] .

The net gain in the active layer $g_{1}$ is found by subtracting a loss term

$$
g_{1}(y)=g(y)-a_{2} N(y)-b_{2}
$$

where $a_{2}$ and $b_{2}$ are constants.

With (9)-(11) the field equation (2) is specified when $N$ and $T$ are known functions of $y$. It is assumed that the temperature is independent of $x$ within the region of interest.

\section{B. Solution of the Field Problem}

The method used for solution of (2) is described in detail in [2] and only the important points will be stated here. The method is a matrix method where the coefficients for an expansion of the solution in Hermite-Grauss functions are found from the eigenvectors and the eigenvalues $\Lambda_{p}$ are the eigenvalues of the matrix.

By the following expansion of the permittivity,

$$
\begin{aligned}
\epsilon_{\text {eff }}(y)-\epsilon_{\text {eff }}(0) & =\left(\epsilon_{\text {eff }}(y)-\epsilon_{\text {eff }}(\infty)\right)-\left(\epsilon_{\text {eff }}(0)-\epsilon_{\text {eff }}(\infty)\right) \\
& =\sum_{n} \alpha_{n} H_{n}\left(\frac{y}{s^{\prime}}\right) \exp \left(-\left(\frac{y}{s^{\prime}}\right)^{2}\right)-\Delta \epsilon_{\text {eff }},
\end{aligned}
$$

all matrix elements are found analytically. $H_{n}$ is the Hermite polynomial of order $n$ and $S^{\prime}$ a scaling length. This expansion is able to give a very precise fit to the calculated profile.

The complex expansion coefficients are found using the orthogonality of Hermite-Gauss functions

$$
\alpha_{n}=\frac{1}{\sqrt{\pi} 2^{n} n ! S^{\prime}} \int_{-\infty}^{\infty} H_{n}\left(\frac{y}{S^{\prime}}\right)\left(\epsilon_{\mathrm{eff}}(y)-\epsilon_{\mathrm{eff}}(\infty)\right) d y .
$$

The expansion is possible if

$$
\left(\epsilon_{\text {eff }}(y)-\epsilon_{\text {eff }}(\infty)\right) \exp \left(+\frac{1}{2}\left(\frac{y}{S^{\prime}}\right)^{2}\right) \longrightarrow 0
$$

$$
\text { for } y \longrightarrow \infty
$$

since this function can then be expanded in Hermite-Gauss functions. The matrix elements can be expressed by $\alpha_{n}$ and the gamua function [2]. With this solution also, mode spacings and far fields are easily found [2].

For the solution of the field problem, $\epsilon(y)$ is often approximated by a parabolic variation, e.g., [5], [6]. This gives directly an analytical solution but it is not possible to obtain a detailed description of $\epsilon$.

In [7] a power series $\epsilon(0)-a^{2} y^{2}+$ higher order terms is used; the first two terms give a Hermite-Gauss solution and the higher order terms give perturbations. This theory will be discussed in Section V.

Also, some special profiles where (2) can be solved analytically have been investigated, e.g., [8]. These profiles, however, are probably of limited interest for detailed calculations. 


\section{Electron Density}

The electron density $N$ is found from the diffusion equation

$$
D \frac{d^{2} N}{d y^{2}}=-\frac{J(y)}{e d}+\frac{N}{\tau_{s}}+\Gamma g(y) \sum_{p} s_{p} I_{p}(y)
$$

where $s_{p}$ is the amplitude and $I_{p}(y)$ is the normalized intensity distribution for mode $p$ given by

$$
I_{p}(y)=\frac{\left|Y_{p}(y)\right|^{2}}{\int_{-\infty}^{\infty}\left|Y_{p}(y)\right|^{2} d y} .
$$

$Y_{p}(y)$ is the solution of (2) for mode $p$. If the lifetime $\tau_{s}$ is independent of $N$ (but not necessarily of $y$ ), (15) can be written as a linear equation using $(10)$ to give

$$
\begin{aligned}
& \frac{d^{2} N}{d y^{2}}=N \cdot A^{\prime}(y)-B^{\prime}(y) \\
& A^{\prime}(y)=\frac{1}{D \tau_{s}}+\frac{\Gamma}{D}\left(a_{1}-a^{\prime}(T(y)-300 K)\right) \sum_{p} s_{p} I_{p}(y) \\
& B^{\prime}(y)=\frac{J(y)}{e d D}+\frac{b_{1} \Gamma}{D} \sum_{p} s_{p} I_{p}(y) .
\end{aligned}
$$

A numerical method for solution of this equation is presented in the Appendix.

The current density $J(y)$ is calculated according to [9]

$$
J(y)= \begin{cases}J_{1} & |y| \leqslant S \\ J_{1} \frac{1}{\left(1+\frac{y-S}{l_{0}}\right)^{2}} & |y|>S .\end{cases}
$$

$J_{1}$ and $l_{0}$ are found from the total current $I$

$$
\begin{aligned}
& J_{1}=\frac{I_{a}^{2}+I(S / L)-I_{a} \sqrt{I_{a}^{2}+2 I(S / L)}}{2 S^{2}} \\
& l_{0}=\frac{I_{a}}{\sqrt{J_{1}}} .
\end{aligned}
$$

The constant $I_{a}$ depends on the thickness $t_{s}$, the resistivity $R_{s}$ of the spreading layer, and the exponent $n k T / e, n \approx 2$, from the $I-V$ characteristic. Thus,

$$
I_{a}=\sqrt{2 \frac{n k T}{e} \frac{t_{s}}{R_{s}}} \text {. }
$$

\section{Temperature}

The temperature variation is found by solving the heat flow equation. In [10] a series expansion for $T(y)$ is given. This result contains the Fourier coefficients for the heat source distribution

$$
a_{n}=\frac{2}{n \pi} \sin \left(b_{n} S\right), b_{n}=\frac{2 n \pi}{A}, \quad n=1,2, \cdots
$$

when a box shaped distribution is assumed. The local thermal resistance becomes

$$
R(y)=\frac{2 S}{A}\left(\frac{t}{\sigma_{\mathrm{act}}}+\frac{t_{4}}{\sigma_{p}}+\frac{t_{6}}{\sigma_{\mathrm{cap}}}\right)+\sum_{n} \begin{gathered}
a_{n} \cos \left(b_{n} y\right) \\
b_{n} \sigma_{\mathrm{act}}\left(r_{1, n}+r_{2, n}\right)
\end{gathered}
$$

where $\sigma$ is the heat conductivity in the layers, and $r_{1, n}$ and $r_{2, n}$ are found from the boundary conditions [10]. The temperature is found by multiplying (22) by the dissipated power per area $1 / 2 S L\left(\hbar \omega / e I-2 P_{\text {out }}\right)$ where $\hbar \omega$ is the photon energy and $P_{\text {out }}$ is the stimulated power emitted at each mirror. In case of pulsed operation this expression is multiplied by a duty cycle factor. This factor can also be used to scale the influence of thermal effects. In this treatment only a heat source in the active layer is considered.

Other heat source distributions can be taken into account by using other Fourier coefficients in (22), but due to the heat flow the exact distribution is not important.

Taking the distribution as a constant for $|y| \leqslant S$ followed by a tail for $|y|>S$ we have a position dependence of the same type as the current density (18). Since the current density falls off slowly we can assume the carrier density and dissipated power to be proportional to $J(Y)$ for $|y| \gg S$, and a convenient description of the thermal resistance is then obtained by replacing $a_{n}$ in (22) with the Fourier coefficients for the current density (18) so that

$$
\begin{aligned}
a_{n}^{\prime}= & \frac{J_{1}}{J_{0}} \frac{2}{n \pi} \sin \left(b_{n} S\right)+\frac{J_{1}}{J_{0}} l_{0}^{2} \frac{4}{A}\left\{\frac{1}{l_{0}} \cos \left(b_{n} S\right)\right. \\
& +b_{n} \cos \left(b_{n} S\right)\left(\cos \left(b_{n} l_{0}\right) \operatorname{si}\left(b_{n} l_{0}\right)-\sin \left(b_{n} l_{0}\right)\right. \\
& \left.\cdot C_{i}\left(b_{n} l_{0}\right)\right)+b_{n} \sin \left(b_{n} S\right)\left(\cos \left(b_{n} l_{0}\right) C_{i}\left(b_{n} l_{0}\right)\right. \\
& \left.\left.+\sin \left(b_{n} l_{0}\right) \operatorname{si}\left(b_{n} l_{0}\right)\right)\right\} \\
J_{0}= & \frac{I}{2 S L}
\end{aligned}
$$

where we have used integral sine and cosine

$$
\begin{aligned}
& s_{i}(x)=-\int_{x}^{\infty} \frac{\operatorname{sint}}{t} d t=-\frac{\pi}{2}+\int_{0}^{x} \frac{\sin t}{t} d t=-\frac{\pi}{2}+S_{i}(x) \\
& C_{i}(x)=-\int_{x}^{\infty} \frac{\text { cost }}{t} d t .
\end{aligned}
$$

\section{E. Lasing Condition}

Spontaneous emission into the lasing modes is neglected; hence, modes at or above the threshold must have gain equal to loss. When the normalized intensity distribution for mode $p$ is

$$
I_{p}(x, y)=I(x) I_{p}(y),
$$

the lasing condition becomes

$$
\int_{-\infty}^{\infty} \int_{-\infty}^{\infty} g(x, y) I(x) I_{p}(y) d x d y=\alpha_{\text {end }}=\frac{1}{L} \ln \left(\frac{1}{R}\right) .
$$

The gain $g$ is given by

$$
g(x, y)= \begin{cases}g_{1}(y) & |x| \leqslant t \\ g_{2}(y)=-\alpha_{\text {out }} & |x|>t .\end{cases}
$$


Using the filling factor $\Gamma(7)$ we get from (26) and (27)

$$
\overline{g_{1 p}}=\frac{\alpha_{\text {end }}+(1-\Gamma) \alpha_{\text {out }}}{\Gamma}=g_{\text {th }}
$$

where the average net gain in the active region for mode $p$ is

$$
\overline{g_{1 p}}=\int_{-\infty}^{\infty} g_{1}(y) I_{p}(y) d y .
$$

The threshold current is defined as the current for which (28) is satisfied for one mode when all amplitude factors $s_{p}$ are zero.

The lasing condition can be written in terms of propagation constants, noting that the propagation in the $z$-direction is described by $e^{-j \beta_{z} z}$. We start from

$$
\beta_{x}(0,0)^{2}+\beta_{y}^{2}+\beta_{z}^{2}=\epsilon_{1}(0) k^{2}
$$

where

$$
\begin{aligned}
\beta_{x}(0,0)^{2} & =\frac{v^{2}}{t^{2}}\left(1-b_{0}-j b_{i}(0)\right) \\
v^{2} & =k^{2} t^{2}\left(\epsilon_{1}(0)-\epsilon_{2}(0)\right) .
\end{aligned}
$$

Using (1), (5), (7), (8b), (27), and (31) we get from (30), when $\operatorname{Re}\left\{\beta_{y}^{2}\right\}$ and high order terms are neglected,

$$
2 \operatorname{Im}\left(\beta_{z}\right\}=g_{1}(0)-\frac{\operatorname{Im}\left\{\beta_{x}(0,0)^{2}\right\}}{n_{\mathrm{eff}}(0) k}-\frac{\operatorname{Im}\left\{\beta_{y}^{2}\right\}}{n_{\mathrm{eff}}(0) k}
$$

where

$$
\frac{\operatorname{Im}\left\{\beta_{x}(0,0)^{2}\right\}}{n_{\text {eff }}(0) k}=g_{1}(0)-g_{1}(0) \Gamma+\alpha_{\text {out }}(1-\Gamma) \text {. }
$$

The two last terms in (32) represent diffraction losses. From the lasing condition

$$
2 \operatorname{Im}\left\{\beta_{z}\right\}=\alpha_{\text {end }}
$$

we finally get for mode $p$

$$
g_{1}(0)-\frac{1}{\Gamma} \frac{\operatorname{Im}\left\{\Lambda_{p}\right\}}{n_{\text {eff }}(0) k}=\frac{\alpha_{\text {end }}+(1-\Gamma) \alpha_{\text {out }}}{\Gamma}
$$

where $\Lambda_{p}$ is the eigenvalue for (2).

\section{Couplings Among Properties}

For a given total current $I$ below threshold, we can find the current density [(18)-(20)], the temperature variation, solve the diffusion equation and then the field equation.

Under lasing conditions the unknowns $N(y), I_{p}(y)$, and $s_{p}$ must be found from (2), (15) and (28), or (35). These equations are coupled via (9)-(11) and (16). This is shown schematically in Fig. 2.

In the model we have not taken into account that the current density may depend on the electron density and temperature variation.

\section{Application of the Model}

Below threshold $\left(\overline{g_{1 p}}<g_{\text {th }}\right.$ for all $\left.p\right)$ the amplitude factors $s_{p}$ are zero so the last term in (15) vanishes, and the electron density can be calculated. From the electron density and

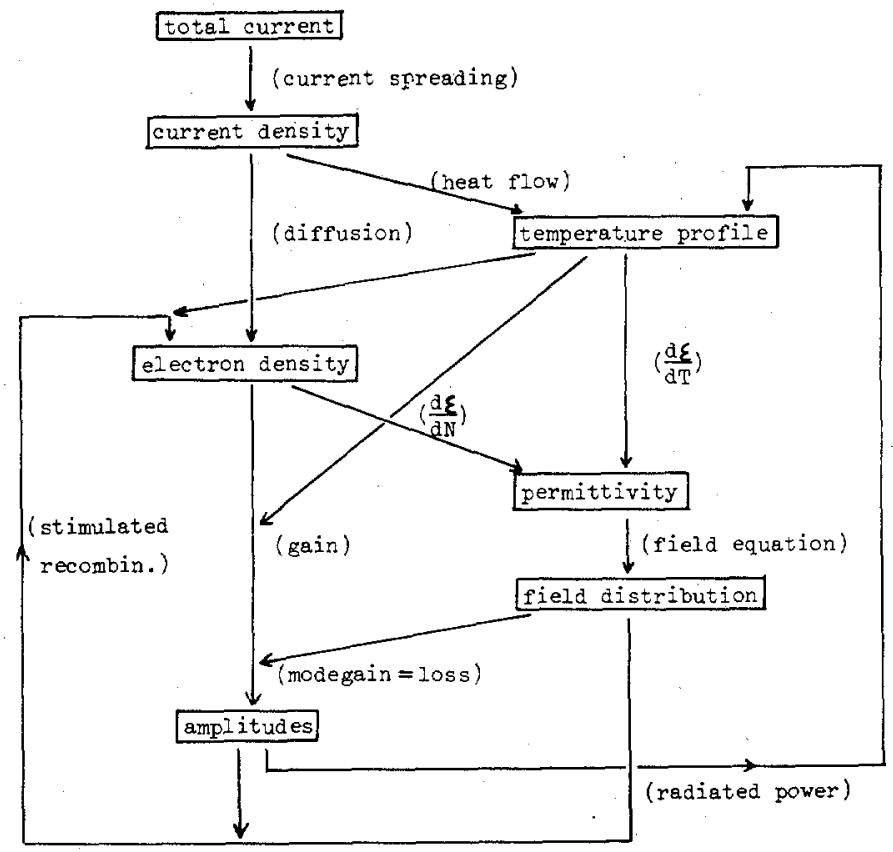

Fig. 2. Graphical representation of the laser model showing properties and couplings taken into account.

temperature the intensity distributions are found. By increasing the current until (28) [or (35)] is satisfied and keeping $s_{p}=0$, we find the threshold current. Examples are given in [2].

For currents above threshold simultaneous solutions to (2), (15) and (28), or (35) are found iteratively. We start by setting $s_{p}=0$ and then find $N(y)$ and $I_{p}(y)$. For currents above threshold we use $I_{p}(y)$ from the previous current value, guess $s_{p}$, and calculate $N(y)$. Keeping $I_{p}(y)$ constant, $s_{p}$ are varied until $N(y)$ found from (15) satisfies (28) for all modes with $s_{p}>0$. Use of (35) during this iteration would require a new calculation of $I_{p}(y)$ for each new set of $s_{p}$ values. The resulting values of $N(y)$ are compared with the original and the calculations are repeated until stable solutions for $N(y)$, $I_{p}(y)$, and $s_{p}$ are found. The routines for the subproblems have all been tested separately on problems with known solutions.

The amplitudes $s_{p}$ are related to the stimulated power per mirror by

$$
P_{\text {out }}=\frac{1}{2} \hbar \omega \ln \left(\frac{1}{R}\right) d \sum_{p} s_{p}
$$

From the calculated amplitudes as a function of the current we get the light current characteristic.

Examples of calculated characteristics are shown in Fig. 3. The various laser parameters used are discussed in [2]. In these examples the power in the fundamental mode saturates at the current value where the next mode starts lasing.

The characteristics have the shape shown in Fig. 4. $I_{t h}$ is the threshold current and $I_{1}$ is the current where the next mode becomes lasing. Between $I_{\text {th }}$ and $I_{1}$ the differential efficiency decreases if improved guiding (e.g., due to temperature) decreases the width of the mode.

From various calculations performed we have obtained the following results. 


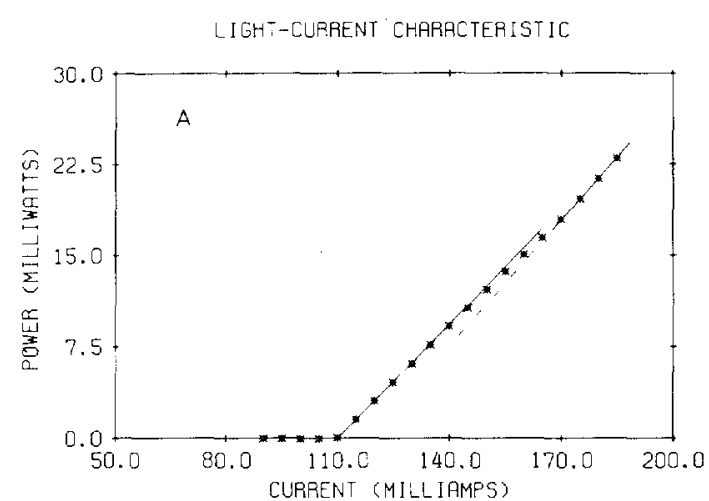

(a)

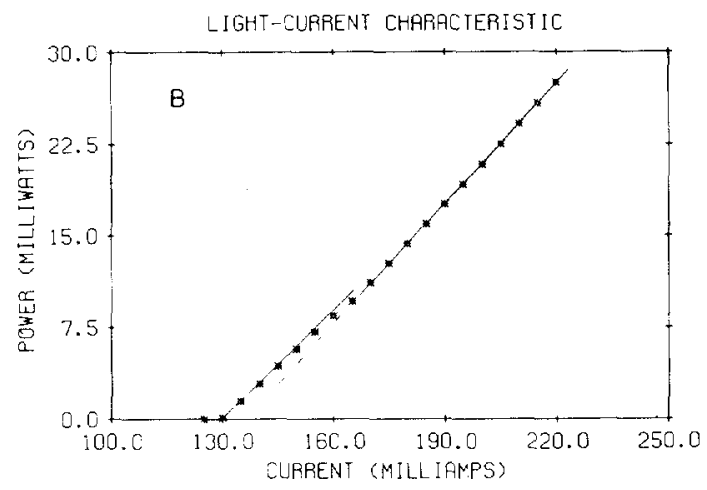

(b)

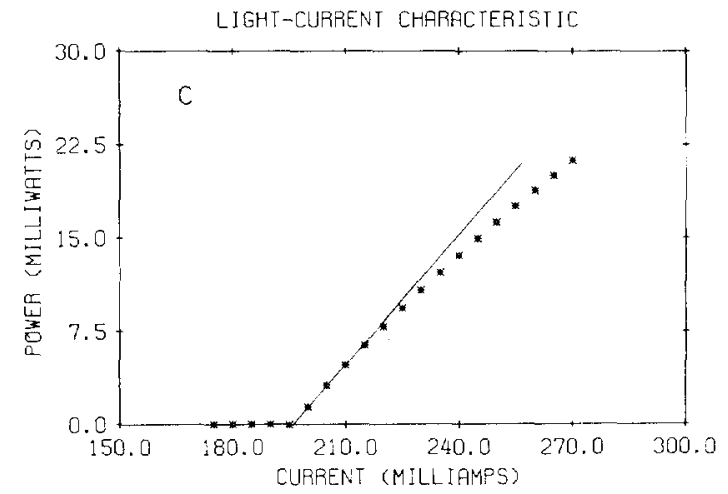

(c)

Fig. 3. Light current characteristics: (a) $S=6 \mu \mathrm{m}, d=0.15 \mu \mathrm{m}$. $S=8 \mu \mathrm{m}, d=0.15 \mu \mathrm{m}$. (c) $S=6 \mu \mathrm{m}, d=0.30 \mu \mathrm{m}$.

1) Increasing stripe width decreases $P_{1}$.

2) For short diffusion lengths the spatial hole burning is stronger and this decreases $P_{1}$.

3) When the length is changed the quantity $\left(I_{1}-I_{t h}\right) / I_{t h}$ remains almost constant. Since $I_{\text {th }}$ decreases faster with decreasing length than the differential efficiency increases, $P_{1}$ will decrease.

4) For thick active layers the thermal guiding is relatively weaker and the carriers give a larger negative real part of $\epsilon_{\text {eff }}$. This reduced guiding will give a higher $P_{1}$.

5) A large number of simulations have been performed using various values of the input parameters. No cases of critical dependence of the result on the input parameters were found.

\section{Discussion}

In contrast to other theoretical treatments of laser properties the present model offers detailed analytical or numerical

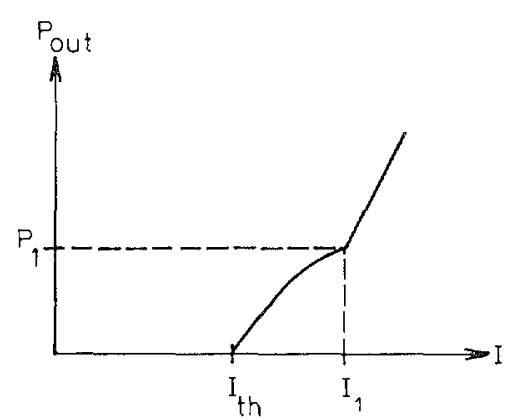

Fig. 4. Typical light current characteristic (exaggerated).

solutions to the subproblems. Particularly, the permittivity and field problems are carefully considered. Multimode operation and thermal effects are also included.

In [7] pure gain guiding is assumed. The gain profile is expanded in a power series and a Hermite-Gauss solution is found from the quadratic term. This solution is perturbed by the higher order terms. The present model has been applied to the parameters used in [7]. The calculated characteristic is almost linear, but with the interesting result that $d^{2} g_{1}(y) /$ $\left.d y^{2}\right|_{y=0}$ becomes zero at the power where the kink in [7] is found. This fact indicates that power expansion combined with perturbation theory is not adequate, and that the kink found in [7] can be explained mathematically: a low value of the quadratic term in the gain expression gives a too broad Hermite-Gauss solution and results in high values of the perturbation terms. This gives "ears" on the intensity distribution which reduce the differential efficiency.

The theory presented in [6] is based on the parabolic approximation. The permittivity is assumed to be a combination of a constant part and a current dependent part. In case of an offset between these two parts the theory predicts both kinks and near-field shifts, as observed experimentally. Furthermore, the theory has the advantage that the calculations can be carried out without a large computer.

In the present paper we have taken advantage of the (ideal) symmetry of the laser structure in order to simplify the calculations; consequently, all calculated near fields are symmetrical. The near fields are always found to be stable and the kinks are associated with higher order modes. Very good agreement with the results for the (slightly asymmetrical) "well behaved" laser reported in [6] is obtained.

The type of kink described in [6] only involves the fundamental mode. For relatively narrow lasers $(2 S \lesssim 15 \mu \mathrm{m})$, this may be the dominating mechanism, since the kinks tend to appear at lower power levels than the kinks calculated by the present model.

\section{Conclusion}

The present model describes the static behavior of $\mathrm{DH}$ lasers in detail. Very few assumptions have been necessary and precise methods are used. By this model it is possible to predict the properties of lasers directly from the technological parameters when the material parameters are known. It will also be possible to optimize the lasers with respect to threshold current, efficiency, linearity, etc.

It is shown that kinks may appear in ideal lasers. 


\section{APPENDIX}

Numerical Solution of the Diffusion Equation

From (17) we see that $A^{\prime}(y)$ is constant and $B^{\prime}(y)$ is zero for large $y$ values. For an ideal symmetrical laser the boundary conditions are

$$
\left.\frac{d N}{d y}\right|_{y=0}=0, \quad N(y) \longrightarrow 0 \text { for } y \longrightarrow \infty .
$$

We consider discrete points

$$
\begin{aligned}
& y_{i}, i=0,1, \cdots, M \\
& y_{0}=0, \quad \Delta y=y_{i+1}-y_{i} \\
& A^{\prime}(y)=A^{\prime}\left(y_{M}\right), \quad B^{\prime}(y)=0 \quad \text { for } y \geqslant y_{M} .
\end{aligned}
$$

We expand the second derivative and use the boundary conditions

$$
\begin{aligned}
\frac{d^{2} N}{d y^{2}} & =\frac{1}{(\Delta y)^{2}}\left(N\left(y_{i+1}\right)-2 N\left(y_{i}\right)+N\left(y_{i-1}\right)\right) \\
\left.\frac{d^{2} N}{d y^{2}}\right|_{y=0} & =\frac{2}{(\Delta y)^{2}}\left(N\left(y_{1}\right)-N\left(y_{0}\right)\right) \\
N\left(y_{M+1}\right) & =N\left(y_{M}\right) e^{-\sqrt{A^{\prime}\left(y_{M}\right)} \Delta y}
\end{aligned}
$$

Equation (17) is transformed into a set of $M+1$ linear equations with the $M+1$ unknowns $N\left(y_{0}\right), N\left(y_{1}\right), \cdots, N\left(y_{M}\right)$. This set of equations can be written

$$
\begin{aligned}
\overline{\bar{C}} \cdot \bar{N}= & \bar{B} \\
\bar{N}= & \left(N\left(y_{0}\right), N\left(y_{1}\right), \cdots, N\left(y_{M}\right)\right) \\
\bar{B}= & (\Delta y)^{2}\left(\frac{1}{2} B^{\prime}\left(y_{0}\right), B^{\prime}\left(y_{1}\right), \cdots, B^{\prime}\left(y_{M-1}\right), 0\right) \\
= & \left(d_{0}, d_{1}, \cdots, d_{M}\right) \\
& \left(\begin{array}{ll}
1+\frac{A^{\prime}\left(y_{0}\right)}{2}(\Delta y)^{2} & r=s=0 \\
2+A^{\prime}\left(y_{r}\right)(\Delta y)^{2} & r=s, s=1, \cdots, M-1 \\
2+A^{\prime}\left(y_{M}\right)(\Delta y)^{2}-e^{-\sqrt{A^{\prime}\left(y_{M}\right)} \Delta y} & r=s=M \\
C_{r S}= & \left\{\begin{array}{l}
r=s-1, s=1, \cdots, M \\
r=s+1, s=0, \cdots, M-1
\end{array}\right. \\
-1 & \text { elsewhere. }
\end{array}\right.
\end{aligned}
$$
many fruitful discussions.

\section{REFERENCES}

[1] G. B. Hocker and W. K. Burns, "Mode dispersion in diffused channel waveguides by the effective index method," Appl. Opt., vol. 16, pp. 113-118, 1977.

[2] J. Buus, "Detailed field model for DH stripe lasers," Opt. Quant. Elec., vol. 10, Nov. 1978.

[3] W. O. Schlosser, "Gain induced modes in planar structures," Bell Syst. Tech. J., vol. 52, pp. 887-905, 1973.

[4] T. L. Paoli, "Waveguiding in a stripe-geometry junction laser," IEEE J. Quantum Electron., vol. QE-13, pp. 662-668, 1977.

[5] A. G. Steventon, P. J. Fiddyment, and D. H. Newman, "Low threshold current proton-isolated (GaAl)As double heterostructure lasers," Opt. Quant. Elec., vol. 9, pp. 519-525, 1977.

[6] G. H. B. Thompson, D. F. Lovelace, and S. E. M. Turley, "Kinks' in the light/current characteristics and near field shifts in (GaAl)As heterostructure stripe lasers and their explanation by the effect of self-focusing on a built-in optical waveguide," Inst. Elec. Eng. Solid-State Elect. Dev., vol. 2, pp. 12-30, 1978.

[7] N. Chinone, "Nonlinearity in power-output-current characteristics of stripe-geometry injection lasers," J. Appl. Phys., vol. 48, pp. 3237-3244, 1977.

[8] W. Streifer, D. R. Scifres, and R. D. Burnham, "Analysis of gain-induced waveguiding in stripe geometry diode lasers," IEEE J. Quantum Electron., vol. QE-14, pp. 418-427, 1978.

[9] W. P. Dumke, "Current thresholds in stripe-contact injection lasers," Solid-State Electron., vol. 16, pp. 1279-1284, 1973, also in W. B. Joyce and S. H. Wemple, "Steady-state junctioncurrent distributions in thin resistive films on semiconductor junction (solutions of $\nabla^{2} v= \pm e^{v}$ ),"J. Appl. Phys., vol. 41, pp. 3818-3830, 1970 .

[10] W. B. Joyce and R. W. Dixon, "Thermal resistance of heterostructure lasers," J. Appl. Phys., vol. 46, pp. 855-862, 1975. 